\title{
Aromaticity of Tyr-202 in the $\alpha 4-\alpha 5$ Loop Is Essential for Toxicity of the Bacillus thuringiensis Cry4A Toxin
}

\author{
Walairat Pornwiroon, Gerd Katzenmeier, Sakol Panyim and Chanan Angsuthanasombat* \\ Laboratory of Molecular Biophysics, Institute of Molecular Biology and Genetics, \\ Mahidol University, Salaya Campus, Nakornpathom 73170, Thailand
}

Received 18 August 2003, Accepted 28 August 2003

\begin{abstract}
The current model for the mechanism of action of the Bacillus thuringiensis Cry $\delta$-endotoxins involves the penetration of the $\alpha 4-\alpha 5$ hairpin into the target midgut epithelial cell membranes, followed by pore formation. In this study, PCR-based mutagenesis was employed to identify a critical residue within the $\alpha 4-\alpha 5$ loop of the 130kDa Cry4A mosquito-larvicidal protein. Alaninesubstitutions of two charged (Asp-198 and Asp-200) and four polar (Asn-190, Asn-195, Tyr-201 and Tyr-202) residues in the $\alpha 4-\alpha 5$ loop were performed. Like the wildtype, all of the mutant toxins were over-expressed as inclusion bodies in Escherichia coli. When $E$. coli cells expressing each mutant toxin were bioassayed against Aedes aegypti larvae, larvicidal activity was completely abolished for the substitution of only Tyr-202, while replacements at the other positions still retained a high level of toxicity. Further replacement of Tyr-202 with an aromatic side chain, phenylalanine, did not affect the toxicity. These results revealed a crucial role in toxin activity for the conserved aromatic residue at the 202 position within the $\alpha 4-\alpha 5$ loop of the Cry $4 A$ toxin.
\end{abstract}

Keywords: Aromaticity, Bacillus thuringiensis, $\delta$-Endotoxin, $\alpha 4-\alpha 5$ loop, Larvicidal activity, Mutagenesis

\section{Introduction}

Bacillus thuringiensis (Bt), a Gram-positive soil bacterium, produces crystalline inclusions during sporulation. These inclusions are composed of one or more proteins, known as $\delta$ endotoxins, that are highly toxic to various insect larvae, and have been widely used as biological insecticides (Aronson $e t$

*To whom correspondence should be addressed.

Tel: 662-441-9003 ext. 1237; Fax: 662-441-9906

E-mail: stcas@mahidol.ac.th al., 1986; Höfte and Whiteley, 1989; Schnepf et al., 1998). The $B t \delta$-endotoxins can be classified into the two families of Cry (crystal) and Cyt (cytolytic) toxins based on the similarity of their deduced amino acid sequences (Höfte and Whiteley, 1989; Crickmore et al., 1998). For example, one of the four major larvicidal proteins that is produced by $B t$ subsp. israelensis (Bti) has been classified as Cry4A, which is highly toxic to mosquito-larvae of Aedes sp., Culex sp., and Anopheles sp. (Höfte and Whiteley, 1989; Schnepf et al., 1998).

Upon ingestion by the susceptible insect larvae, the $B t$ crystalline inclusions are solubilized under the alkaline conditions of the larval midgut and are proteolytically activated by gut proteases to release the active toxins (Höfte and Whiteley, 1989). For several Cry toxins, the activated toxins bind to specific receptors that are located on the brush border membrane of midgut epithelial cells (Schnepf et al., 1998; de Maagd et al., 2001). Subsequently, the toxins insert into the membrane to form leakage pores that cause the cell to swell and lyse by colloid-osmotic lysis (Knowles and Ellar, 1987). However, the precise mechanism of action at the molecular level of $B t$ toxins is still not completely understood.

The X-ray crystal structures of four different Cry toxins, Cry1Aa (Grochulski et al., 1995) Cry2Aa (Morse et al., 2001), Cry3Aa (Li et al., 1991), and Cry3Bb (Galitsky et al., 2001) display a high degree of overall structural similarity and are composed of three distinct domains. The N-terminal domain $I$ is a seven $\alpha$-helix bundle in which the helix 5 is relatively hydrophobic and completely surrounded by six other amphipatic helices. A function for this domain in the membrane insertion and pore formation is supported by a number of studies that demonstrate the isolated helical fragments, $\alpha 1-\alpha 7$ of Cry3B2 and Cry1Ac (Walters et al., 1993; Von Tersch et al., 1994) or $\alpha 1-\alpha 5$ of Cry4B (Puntheeranurak et al., 2001; 2004) that are responsible for the pore-formation and generation of ion-selective channels.

The "umbrella model" has been proposed to describe the toxicity mechanism of the Bt Cry toxins that involves an 
insertion of helices 4 and 5 into the membrane as a helical hairpin structure, with the remaining helices lying on the membrane surface (Gazit et al., 1998). This model is supported by a number of studies that demonstrate the crucial role of $\alpha 4$ and $\alpha 5$ in the toxicity of different Cry toxins. The results of in vitro studies suggest that $\alpha 4$ lines the lumen of the pore and participates in ion-channel formation, while the hydrophobic $\alpha 5$ interacts with the lipid membranes and participates in toxin oligomerisation (Schwartz et al., 1997; Masson et al., 1999; Nunez-Valdez et al., 2001). Furthermore, membrane permeation studies with Cry1Ac have revealed that the $\alpha 4$-loop- $\alpha 5$ segment is more active in membrane permeation than either the isolated helices or their mixtures, suggesting that the presence of the loop-structure is a requirement for efficient insertion of the toxin into the membrane (Gerber and Shai, 2000).

In previous studies, we demonstrated that $\alpha 4$ and $\alpha 5$ of the $130-\mathrm{kDa}$ Bti Cry4B toxin are important determinants of toxicity, likely being involved in membrane insertion and pore formation (Uawithya et al., 1998; Sramala et al., 2000). In addition, the specific structure for the positively-charged sidechain of Arg-158 in $\alpha 4$ was found to play a pivotal role in the Cry4B larvicidal activity (Sramala et al., 2001). We further demonstrated that the polar residue at position-166 and the aromatic residue at position-170 within the $\alpha 4-\alpha 5$ loop are important structural determinations for the toxicity of this toxin (Kanintronkul et al., 2003). In this report, we showed that an analogous effect on mosquito-larvicidal activity of the closely-related 130-kDa Bti toxin, Cry4A, was observed when charged or polar residues in the $\alpha 4-\alpha 5$ loop were altered. The results reveal that Tyr-202, which is very conserved among the Cry toxins, is critically involved in the Cry4A toxicity, supporting the notion that an aromatic structure of the highlyconserved tyrosine residue within the $\alpha 4-\alpha 5$ loop is an essential prerequisite for the toxic action of the Cry $\delta$ endotoxins.

\section{Materials and Methods}

Construction of mutant toxins The recombinant plasmid pMEx-B4A encoding the $130-\mathrm{kDa} B t$ Cry4A larvicidal protein, which has been reconstructed in the pMEx8 expression vector (Buttcher et al., 1990) under control of the tac promoter together with the cry $4 B$ promoter (Angsuthanasombat et al., 1987), was used as a template for the site-directed mutagenesis. Mutant plasmids were generated by a polymerase chain reaction (PCR) using a pair of mutagenic primers (Table 1) that were purchased from Proligo Inc. (Singapore) and $P f u$ DNA polymerase, following the procedure of the QuickChange ${ }^{\mathrm{TM}}$ Mutagenesis Kit (Stratagene, La Jolla, USA). The mutant clones with the required mutation were first identified by restriction endonuclease digestion of the plasmids, and verified by DNA sequencing, using a BigDye ${ }^{\mathrm{TM}}$ Terminator Cycle Sequencing Kit (Perkin-Elmer, Norwalk, USA).

Expression and solubilisation of toxin inclusions Each $E$. coli
JM109 clone that harbored the wild-type plasmid or its mutants was grown at $30^{\circ} \mathrm{C}$ in a Luria-Bertani medium containing $100 \mu \mathrm{g} \mathrm{mL}^{-1}$ ampicillin until $\mathrm{OD}_{600}$ of the culture reached 0.3-0.5. Toxin expression was induced with isopropyl- $\beta$-D-thiogalactopyranoside (IPTG) at a final concentration of $0.1 \mathrm{mM}$ for $10 \mathrm{hrs}$, and subsequently analyzed by sodium dodecyl sulfate-(10\% w/v) polyacrylamide gel electrophoresis (SDS-PAGE). The cells were harvested by centrifugation and resuspended in cold distilled water. The cell suspension was lysed in a French Pressure Cell at 10,000 psi. The cell lysate was centrifuged at $8,000 \times g, 4^{\circ} \mathrm{C}$ for $15 \mathrm{~min}$. The pellets were washed 3 times in cold distilled water and suspended by sonication. Protein concentrations of the partialpurified inclusions were determined by using a protein microassay reagent (Bio-Rad, Hercules, USA) with bovine serum albumin fraction V (Sigma, St. Louis, USA) as the standard. Protoxin

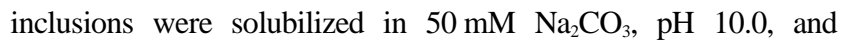
incubated at $37^{\circ} \mathrm{C}$ for $1 \mathrm{hr}$, as previously described (Uawithya et al., 1998). After centrifugation at $8,000 \times g$ for $10 \mathrm{~min}$, the supernatants were analyzed by SDS-PAGE and compared with the inclusion suspension.

Mosquito-larvicidal activity assays Bioassays were performed as previously described (Sramala et al., 2001), using 2-day old Aedes aegypti larvae that was reared from eggs that were obtained from the mosquito-rearing facility of the Institute of Molecular Biology and Genetics, Mahidol University, Thailand. Both the rearing and bioassays were performed at room temperature $\left(25^{\circ} \mathrm{C}\right)$. Assays were carried out in $1 \mathrm{~mL}$ of E. coli suspension $\left(10^{8}\right.$ cells suspended in distilled water) in a 48-well microtitre plate (11.3-mm

Table 1. Complementary primers for substituting a code residue with different amino acids

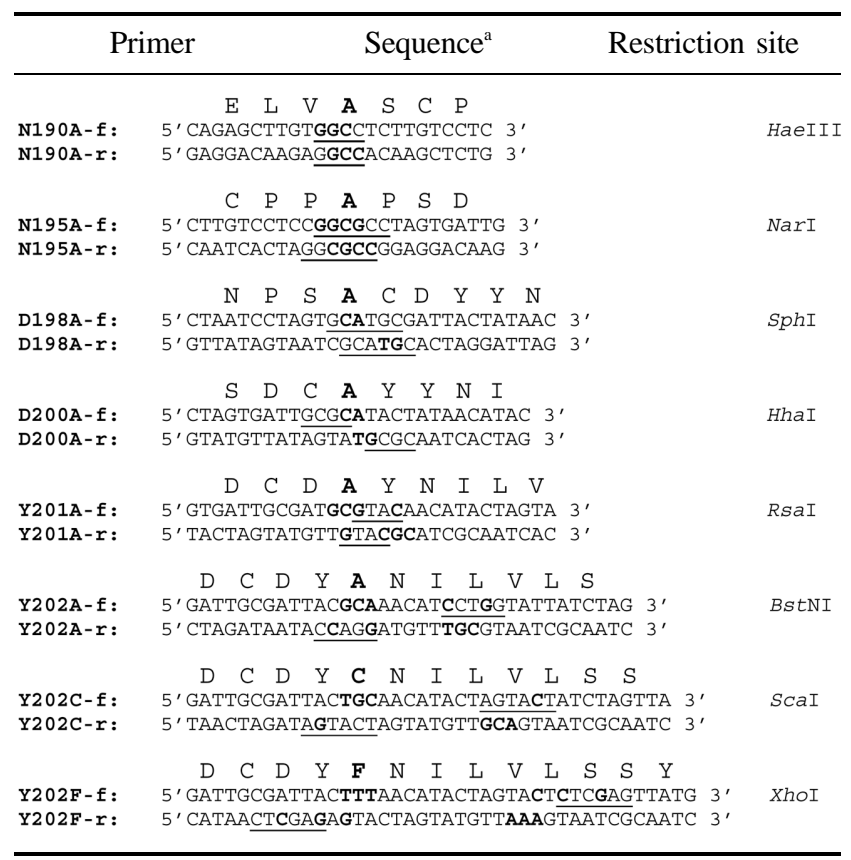

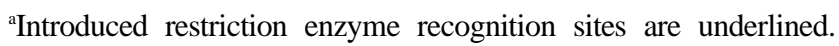
Mutated nucleotide residues are shown in boldface. Deduced amino acid sequences are shown above of each pair of oligonucleotide primers. 
well diameter, Costar, USA) with 10 larvae per well and a total of 100 larvae for each type of E. coli samples. E. coli cells containing the pMEx 8 vector were used as a negative control. Mortality was recorded after a 24 -hr incubation period.

\section{Results and Discussion}

Based on multiple sequence alignments of the known Bt Cry toxin structures and the homology-based Cry4 models (Angsuthanasombat et al., 2004), the interhelical loop connecting $\alpha 4$ and $\alpha 5$ of the Cry4A toxin is composed of sixteen amino acids with the majority being polar and charged residues (Fig. 1a). Previously, we demonstrated that polarity and aromaticity for Asn-166 and Tyr-170, respectively, in the $\alpha 4-\alpha 5$ loop are critically involved in the larvicidal activity of the Cry4B toxin (Kanintronkul et al., 2003). In the present study, we constructed several mutants in the $\alpha 4-\alpha 5$ loop region of Cry4A in order to determine the residue that is responsible for the toxin activity. Two negatively-charged (Asp-198 and Asp-200) and four polar (Asn-190, Asn-195, Tyr-201 and Tyr-202) residues were initially selected for the substitution with alanine via PCR-based directed mutagenesis. The loop mutant toxins were expressed in E. coli under inducible control of the tac promoter. Upon addition of IPTG to the mid-exponential phase cultures, all of the mutant Cry4A protoxins were predominantly produced in the form of sedimentable inclusion bodies. When the E. coli lysates were analyzed by SDS-PAGE, the protein expression levels for all of the mutants were comparable to those of the wild-type (data not shown).

To assess the solubility of the mutant protoxin inclusions in comparison with that of the wild-type, experiments were conducted using carbonate buffer, $\mathrm{pH} 10.0$. The amount of the $130-\mathrm{kDa}$ Cry4A soluble proteins in the supernatant were compared with those of the proteins that were initially used in order to determine the percentage of toxin solubilisation. Figure 2 shows that the toxin inclusions of the N190A, D198A, D200A, and Y201A mutants were soluble in this buffer, giving approximately $60-70 \%$ solubility. This is comparable to the solubility of the wild-type inclusions under similar conditions. On the other hand, a nearly complete loss of the inclusion solubility was observed for the two remaining mutants, N195A and Y202A. However, toxin inclusions of the two closely-related loop-Cry4B mutants, N166A and Y170A as mentioned previously, were found to be relatively soluble in this buffer (Kanintronkul et al., 2003). At this stage, the reason for this difference in solubility between the two loopmutants of Cry4A and Cry4B is unclear. It does, however, lead to the interesting possibility that single-alanine

(A)
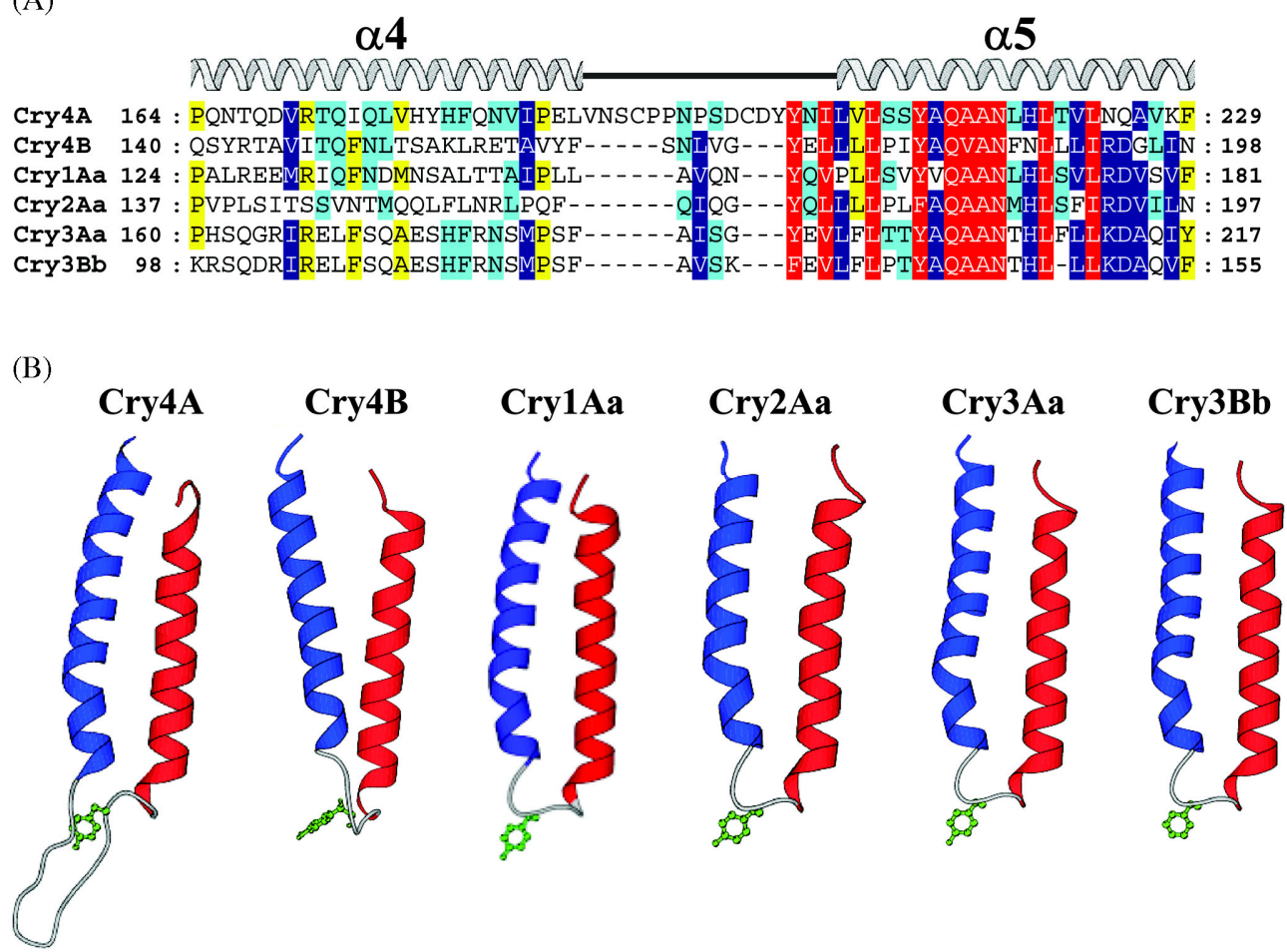

Fig. 1. (A) Multiple sequence alignment of the transmembrane $\alpha 4$-loop- $\alpha 5$ fragment of Cry $4 \mathrm{~A}$ with those of the crystal structures of Cry1Aa, Cry2Aa, Cry3Aa, and Cry3Bb toxins and the homology-based Cry4B model. The sequences were aligned using the Clustal X Program. The corresponding $\alpha 4$-loop- $\alpha 5$ is shown above the sequences. (B) Side views of the $\alpha 4$-loop- $\alpha 5$ helical hairpins of the homology-based Cry4A and Cry4B models, and the known structures of Cry1Aa, Cry2Aa, Cry3Aa, and Cry3Bb. Blue and red ribbons represent $\alpha 4$ and $\alpha 5$, respectively. The conserved loop-residue, Tyr, is shown in ball-and-stick in all six helical hairpins. 

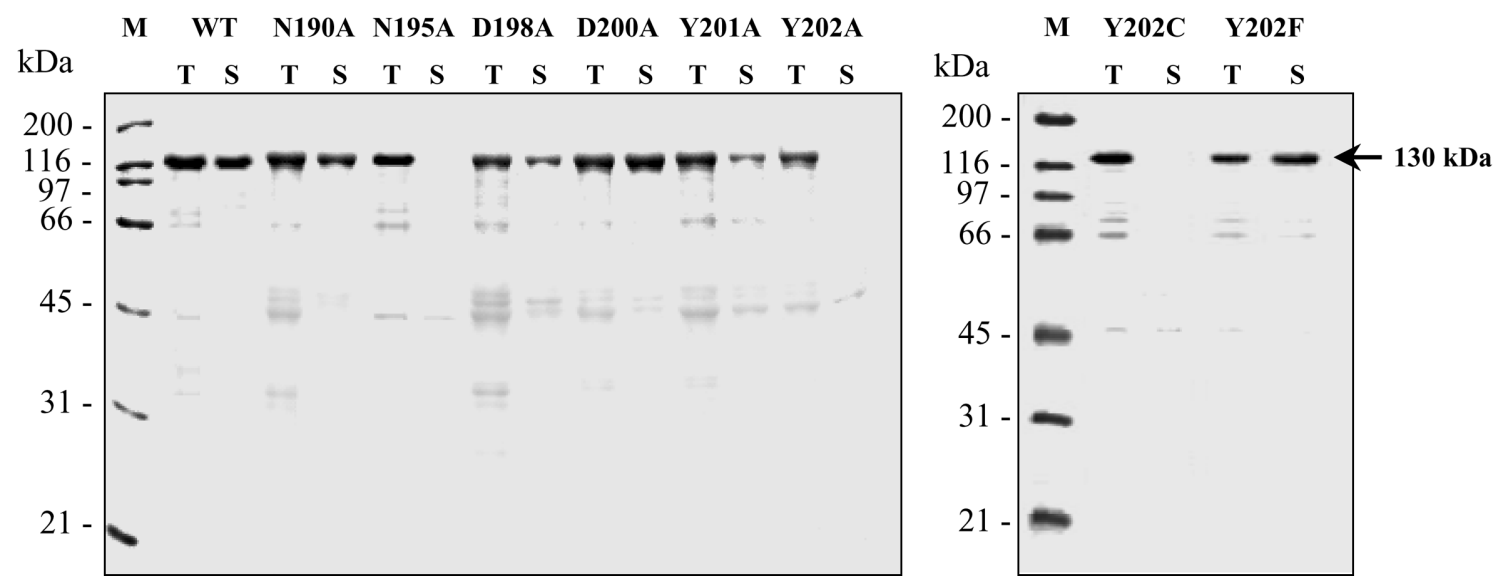

Fig. 2. SDS-PAGE (Coomassie brilliant blue-stained $10 \%$ gel) analysis of the partially-purified 130-kDa protein inclusions that were extracted from E. coli expressing the wild-type (WT) and Cry4A mutant toxins and solubilized in a carbonate buffer, $\mathrm{pH} 10.0$. (T) and (S) represent total fractions and an equivalent volume of the supernatants after centrifugation, respectively. (M) represents the molecular mass standards.

substitutions at Asn-195 and Tyr-202 of the Cry4A toxin could disturb the structural characteristics that consequently affect the toxin-inclusion formation, as shown by a drastic decrease in solubility.

To determine the effect of the $\alpha 4-\alpha 5$ loop mutations on the Cry4A bioactivity, E. coli cells expressing each mutant toxin were tested for their biological activity towards $A$. aegypti mosquito-larvae. Replacement at only Tyr-202 with alanine almost completely abolished larvicidal activity, whereas alanine-substitutions at the other positions (Asn-190, Asn195, Asp-198, Asp-200 and Tyr-201) did not affect the Cry4A toxicity. Further analysis via specific mutations revealed that the conversion of this critical tyrosine residue to cysteine resulted in a drastic loss of toxicity, but replacement with the aromatic residue (i.e. phenylalanine) retained the high level of larvicidal activity (Fig. 3). The level of the protein expression of both the Y202C and Y202F mutant toxins was approximately the same as that of the wild-type. These results, together with the highly structural conserved level of the tyrosine residue at this position among the Cry toxins (Fig. 1b), suggest the essential feature of an aromatic structure at this critical position for the toxin activity. The data further support our previous findings that Tyr-170 in the $\alpha 4-\alpha 5$ loop plays an important role in the larvicidal activity of the 130$\mathrm{kDa}$ Cry4B toxin, since substitutions with only the aromatic residues (i.e. Phe or Trp) were shown to restore bioactivity towards mosquito-larvae (Kanintronkul et al., 2003).

For in vitro solubility, like the Y202A mutation, the substitution of Tyr-202 with Cys reduced the solubility in vitro of toxin inclusions, while a conversion to Phe still exhibited the same solubility characteristics as that of the wild-type (Fig. 2). Although insolubility of the toxin inclusions and the loss of toxicity are seemingly correlated for both the Y202A and Y202C mutants, the inclusion solubility in vitro may not necessarily reflect toxin activity in vivo, as observed for the N195A mutant which was insoluble in the carbonate buffer,

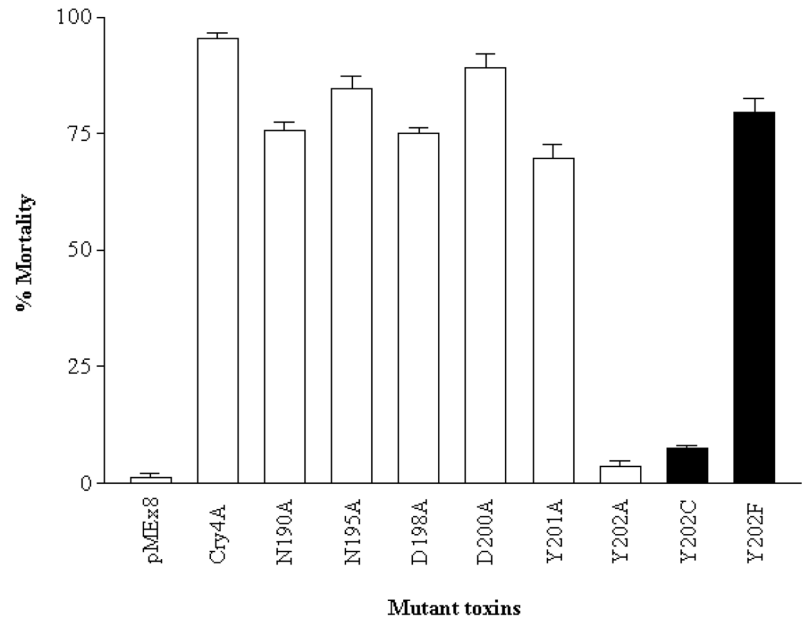

Fig. 3. Mosquito-larvicidal activities of $E$. coli cells expressing the Cry4A wild-type or its mutant toxins (N190A, N195A, D198A, D200A, Y201A, Y202A, Y202C, and Y202F) against A. aegypti larvae. Error bars indicate standard errors of the mean from three independent experiments.

but still bioactive (Figs. 2 and 3). It has been demonstrated that the single-proline substitution in $\alpha 6$ of Cry4B dramatically perturbed the inclusion dissolvability, but did not affect its larvicidal activity (Sramala et al., 2000). Also, the detected difference in solubilisation in vitro for the cloned Cry4A toxin inclusions, which were purified form two different $B t$ recipient strains, is not a factor for toxicity in vivo (Angsuthanasonbat et al., 1992). Presumably, the larval gut proteases in vivo might facilitate the dissolution of the ingested toxin inclusions that would negate the differences between the observed larvicidal activities of the bioactive N195A and non-active Y202A or Y200C mutants.

Studies with several membrane proteins have indicated that the aromatic residues are predominantly found at or near the 
lipid-water interface (Ulmschneider and Sansom, 2001). These aromatic residues have been proposed to function in anchoring the proteins into the membrane through interactions of their aromatic rings with phospholipid head groups (Yau et al., 1998; Killian and Von Heijne, 2000), maintaining rigidity in the periphery of the transmembrane segments (Tsang and Saier, 1996), allowing vertical mobility of the transmembrane helical region with respect to the membranes (Schiffer and Deber, 1990), facilitating translocation of the periplasmic portion of proteins through the membrane, thereby acting as determinants of protein orientation (Schiffer et al., 1992). Taken together, a function for Tyr-202 in the $\alpha 4-\alpha 5$ loop of the Cry4A toxin may conceivably be an interaction with the phospholipid head groups for stabilizing the oligomeric pore structure.

In conclusion, this study demonstrates that the aromaticity of Tyr-202 in the $\alpha 4-\alpha 5$ loop plays a crucial role in the Cry $4 \mathrm{~A}$ toxicity that further supports the notion that the aromatic structure of the highly-conserved tyrosine residue within the loop connecting the two transmembrane helices, $\alpha 4$ and $\alpha 5$, is essential for toxic action of the Bt Cry $\delta$-endotoxins. However, further studies are still required to elucidate the exact role of this critical residue in toxin function.

Acknowledgments This work was supported by the grant number BRG/08/2544 from the Thailand Research Fund. A Royal Golden Jubilee Ph.D. research scholarship to W.P. is gratefully acknowledged. We are also grateful to Ms. Somsri Sakdee and Ms. Chaweewan Shimwai for their technical assistance.

\section{References}

Angsuthanasombat, C., Chungjatupornchai, W., Kertbundit, S., Luxananil, P., Settasatian, C., Wilairat, P. and Panyim, S. (1987) Cloning and expression of $130-\mathrm{kDa}$ mosquito-larvicidal $\delta$-endotoxin gene of Bacillus thuringiensis var israelensis in Escherichia coli. Mol. Gen. Genet. 208, 384-389.

Angsuthanasombat, C., Crickmore, N. and Ellar, D. J. (1992) Comparison of Bacillus thuringiensis subsp. israelensis CryIVA and CryIVB cloned toxins reveals synergism in vivo. FEMS Microbiol. Lett. 94, 63-68.

Angsuthanasombat, C., Uawithya, P., Leetachewa, S., Pornwiroon, W., Ounjai, P., Kerdcharoen, T., Katzenmeier, G. and Panyim, S. (2004) Bacillus thuringiensis Cry4A and Cry4B mosquitolarvicidal proteins: homology-based 3D model and implications for toxin activity. J. Biochem. Mol. Biol. 37, 304-313.

Aronson, A. I., Beckman, W. and Dunn, P. (1986) Bacillus thuringiensis and related insect pathogens. Microbiol. Rev. 50, 1-24.

Buttcher, V., Ruhlmann, A. and Cramer, F. (1990) Improved single-stranded DNA producing expression vectors for protein manipulation in Escherichia coli. Nucleic Acids Res. 18, 1075.

Crickmore, N., Zeigler, D. R., Feitelson, J., Schnepf, E., Van Rie, J., Lereclus, D., Baum, J. and Dean, D. H. (1998) Revision of the nomenclature for the Bacillus thuringiensis pesticidal crystal proteins. Microbiol. Mol. Biol. Rev. 62, 807-813.

Galitsky, N., Cody, V., Wojtczak, A., Ghosh, D., Luft, J. R., Pangborn, W. and English, L. (2001) Structure of the insecticidal bacterial delta-endotoxin Cry3Bb1 of Bacillus thuringiensis. Acta Crystallogr. D. Biol. Crystallogr. 57, 11011109.

Gerber, D. and Shai, Y. (2000) Insertion and organization within membranes of the delta-endotoxin pore-forming domain, helix 4-loop-helix 5 , and inhibition of its activity by a mutant helix 4 peptide. J. Biol. Chem. 275, 23602-23607.

Grochulski, P., Masson, L., Borisova, S., Pusztai-Carey, M., Schwartz, J.-L., Brousseau, R. and Cygler, M. (1995) Bacillus thuringiensis CryIA(a) insecticidal toxin: crystal structure and channel formation. J. Mol. Biol. 254, 447-464.

Höfte, H. and Whiteley, H. R. (1989) Insecticidal crystal proteins of Bacillus thuringiensis. Microbiol. Rev. 53, 242-255.

Kanintronkul, Y., Sramala, I., Katzenmeier, G., Panyim, S. and Angsuthanasombat, C. (2003) Specific mutations within the $\alpha 4-\alpha 5$ loop of the Bacillus thuringiensis Cry4B toxin reveal a crucial role of Asn-166 and Tyr-170. Mol. Biotechnol. 24, 1119.

Killian, J. A. and Von Heijne, G. (2000) How proteins adapt to membrane-water interface. Trends Biochem. Sci. 25, 429-434.

Knowles, B. H. and Ellar, D. J. (1987) Colloid-osmotic lysis is a general feature of the mechanism of action of Bacillus thuringiensis delta endotoxin with difference insect specificity. Biochim. Biophys. Acta 924, 509-518.

Li, J., Carroll, J. and Ellar, D. J. (1991) Crystal structure of insecticidal delta-endotoxin from Bacillus thuringiensis at $2.5 \AA$ resolution. Nature 353, 815-821.

Masson, L., Tabashnik, B. E., Liu, Y. B., Brousseau, R. and Schwartz, J.-L. (1999) Helix 4 of the Bacillus thuringiensis Cry1Aa toxin lines the lumen of the ion channel. J. Biol. Chem. 274, 31996-32000.

Morse, R. J., Yamamoto, T. and Stroud, R. M. (2001) Structure of Cry2Aa suggests an unexpected receptor binding epitope. Structure (Camb.) 9, 409-417.

Nunez-Valdez, M.-E., Sanchez, J., Lina, L., Guereca, L. and Bravo, A. (2001) Structural and functional studies of $\alpha$-helix 5 region from Bacillus thuringiensis Cry $1 \mathrm{Ab} \delta$-endotoxins. Biochimi. Biophys. Acta 1546, 122-133.

Pawagi, A. B. and Deber, C. M. (1990) Ligand-dependent quenching of tryptophan fluorescence in human erythrocyte hexose transport protein. Biochemistry 29, 950-955.

Puntheeranurak, T., Leetachewa, S., Katzenmeier, G., Krittanai, C., Panyim, S. and Angsuthanasombat, C. (2001) Expression and biochemical characterization of the Bacillus thuringiensis Cry4B $\alpha 1-\alpha 5$ pore-forming fragment. J. Biochem. Mol. Biol. 34, 293-298.

Puntheranurak, T., Uawithya, P., Potvin, L., Angsuthanasombat, C., and Schwartz, J.-L. (2004) Ion channels formed in planar lipid bilayers by the dipteran-specific Cry4B Bacillus thuringiensis toxin and its $\alpha 1-\alpha 5$ fragment. Mol. Membr. Biol. 21, 67-74.

Schiffer, M., Chang, C. H. and Stevens, F. J. (1992) The functions of tryptophan residues in membrane proteins. Protein Eng. 5, 213-214.

Schnepf, E., Crickmore, N., Van Rie, J., Lereclus, D., Baum, J., Feitelson, J., Zeigler, D. R. and Dean, D. H. (1998) Bacillus thuringiensis and its pesticidal crystal proteins. Microbiol. Mol. Biol. Rev. 62, 775-806. 
Schwartz, J.-L., Juteau, M., Grochulski, P., Cygler, M., Prefontaine, G., Brousseau, R. and Masson, L. (1997) Restriction of intramolecular movements within the Cry1Aa toxin molecule of Bacillus thuringiensis through disulfide bond engineering. FEBS Lett. 410, 397-402.

Sramala, I., Leetachewa, S., Krittanai, C., Katzenmeier, G., Panyim, S. and Angsuthanasombat, C. (2001) Charged residues screening in helix 4 of the Bacillus thuringiensis Cry4B toxin reveals one critical residue for larvicidal activity. J. Biochem. Mol. Biol. Biophys. 5, 219-225.

Sramala, I., Uawithya, P., Chanama, U., Leetachewa, S., Krittanai, C., Katzenmeier, G., Panyim, S. and Angsuthanasombat, C. (2000) Single proline substitutions of selected helices of the Bacillus thuringiensis Cry4B toxin affect inclusion solubility and larvicidal activity. J. Biochem. Mol. Biol. Biophys. 4, 187193.

Tsang, S. and Saier, M. H., Jr. (1996) A simple flexible program for the computational analysis of amino acyl residue distribution in proteins: application to the distribution of aromatic versus aliphatic hydrophobic amino acids in transmembrane alpha-helical spanners of integral membrane transport proteins. J. Comput. Biol. 3, 185-190.

Uawithya, P., Tuntitippawan, T., Katzenmeier, G., Panyim, S. and Angsuthanasombat, C. (1998) Effects on larvicidal activity of single proline substitutions in alpha 3 or alpha 4 of the Bacillus thuringiensis Cry4B toxin. Biochem. Mol. Biol. Int. 44, 825-832.

Ulmschneider, M. B. and Sansom, M. S. P. (2001) Amino acid distributions in integral membrane protein structures. Biochimi. Biophys. Acta 1512, 1-14.

Von Tersch, M. A., Slatin, S. L., Kulesza, C. A. and English, L. H. (1994) Membrane-permeabilizing activities of Bacillus thuringiensis coleopteran-active toxin CryIIIB2 and CryIIIB2 domain I peptide. Appl. Environ. Microbiol. 60, 3711-3717.

Walters, F. S., Slatin, S. L., Kulesza, C. A. and English, L. H. (1993) Ion channel activity of N-terminal fragments from CryIA(c) delta-endotoxin. Biochem. Biophys. Res. Commun. 196, 921-926.

Yau, W. M., Wimley, W. C., Gawrisch, K. and White, S. H. (1998) The preference of tryptophan for membrane interfaces. Biochemistry 37, 14713-14718. 